\title{
Read clinical terms and child health
}

\author{
David A Walker, Katie Thomson
}

The development of community and hospital based clinical information technology applications is an ever expanding process. The complexity of such systems ranges from stand alone clinical audit/research databases through to hospital wide integrated systems covering the majority of processes involved in patient care. Whenever such systems are introduced, the need to identify groups of patients with common factors such as diagnoses or operations is central to the success of the system. At this stage, those involved in their installation have to grapple with the problem of standardising terminology to describe these elements of the clinical record. They have to ensure that the process of data entry is accurate and consider ways that the system can be made to comply with the NHS standards for the measurement of hospital activity.

A common solution is to adopt standardised classifications of diagnoses or procedures such as the International Classification of Diseases (ICD) or that of the Office of Population Censuses and Surveys (OPCS). Such classifications have the advantage of being nationally or internationally agreed and each term has an associated alpha numerical code suitable for rapid processing by computer. They have the disadvantage of being large and unwieldy in every day use, they use terminology that is often not current, and they have not, until recently, been computerised. These difficulties have been recognised by the NHS information management executive who are proposing a national computer network to share patient information between health care units. The same question of health care terminology and associated standardisation of codes is central to this major national health objective. ${ }^{12}$

Department of Child

Health, University

Hospital, Queen's

Medical Centre,

Nottingham NG7 2UH

D A Walker

Child Health Specialty

Working Group,

Clinical Terms

Project, NHS Centre

for Coding and

Classification,

Loughborough

K Thomson

Correspondence to:

Dr Walker.

\section{Read clinical terms}

Clinical information systems have been in use most widely and for the longest period by general practitioners in the UK. The need for standardised terminology was addressed by Dr James Read, a general practitioner from Loughborough. He started to collect and code health terms relevant to general practice as well as to develop the software that allowed these terms to be selected and incorporated into computerised medical records. From these small beginnings, the thesaurus of Read clinical terms, as they are now called, was developed. These terms and their codes have been adopted by the NHS as the standardised computer language for health care and have been made crown copyright. ${ }^{3-5}$ The sale of the licence to use the codes as well as the provision of facilities for training in their use is the function of Computer Aided Medical Systems (CAMS; Tannery Buildings, 58-60 Woodgate, Loughborough, Leics LE11 2TQ), who trade under a government agreement. Software already exists that permits the selection of terms and their translation into any code compatible with existing international health classifications including OPCS, ICD 9, ICD 10, and ICD $O$.

\section{The clinical terms project}

The thesaurus of Read clinical terms are now being further developed and expanded to meet the requirements of hospital based systems. This process is being carried out by a national 'clinical terms project'. The clinical terms project is funded by the Department of Health and coordinated by the NHS Centre for Coding and Classification (NHS CCC) in Loughborough. The project was launched in April 1992 and ran until April 1994 by which time an expanded thesaurus of health terms was produced and coded within the Read system. The project consists of 54 specialty working groups representing all the branches of medicine and surgery. The child health specialty working group is responsible for terms relating to community child health, general and specialty paediatrics, and paediatric surgery. Additional specialty working groups have been established to represent nursing and midwifery and other professions allied to medicine such as the 'therapies', dentistry, podiatry, and chiropody. ${ }^{6}$

\section{British Paediatric Association (BPA)} Diagnostic Codes Working Party

The BPA obtained funds from the Department of Health in 1990 to review the diagnostic terminology within the new version of the International Classification of Diseases (ICD 10) and to publish a paediatric subset of diagnostic terms and codes compatible with ICD 10. This project was overseen by the BPA Diagnostic Codes Working Party which was chaired by Dr Keith Dodd. The project research worker was Dr Paul Crawshaw and specialist advice was provided by representatives from 
Read clinical term structure

Structure of Read codes

Preferred term: Diabetes mellitus

Synonym: Sugar diabetes

Abbreviation: DM

\begin{tabular}{|c|c|c|c|}
\hline \multicolumn{4}{|c|}{ ncing } \\
\hline Read & Clinical term ( 30 and 60 character term shown) & ICD-9 & $I C D-10$ \\
\hline $\begin{array}{l}\text { B200 } \\
\text { B2001 }\end{array}$ & $\begin{array}{l}\text { Diabetes mellitus } \\
\text { Diabetes mellitus with no complications } \\
\text { DM with no complication }\end{array}$ & $\begin{array}{l}250 \\
250 \cdot 0\end{array}$ & $\begin{array}{l}\text { E10-E14 } \\
\text { E13 }\end{array}$ \\
\hline B2002 & $\begin{array}{l}\text { Diabetes mellitus with neuropathy } \\
\text { DM with neuropathy }\end{array}$ & $250 \cdot 3$ & E13 \\
\hline B2003 & $\begin{array}{l}\text { Diabetes mellitus with nephropathy } \\
\text { DM with nephropathy }\end{array}$ & $250 \cdot 5$ & E13 \\
\hline B2004 & $\begin{array}{l}\text { Insulin dependent diabetes mellitus } \\
\text { Insulin dependent DM }\end{array}$ & 250 & E10 \\
\hline B2005 & $\begin{array}{l}\text { Non-insulin dependent diabetes mellitus } \\
\text { Non-insulin dependent DM }\end{array}$ & 250 & E11 \\
\hline B2006 & $\begin{array}{l}\text { Malnutrition related diabetes mellitus } \\
\text { Malnutrition related DM }\end{array}$ & 250 & E12 \\
\hline
\end{tabular}

each of the BPA specialty groups. The outcome from this project is in the process of being prepared for publication by the BPA and should be available in a written format at the time ICD 10 is formally launched. It has also formed a vital part of the child health specialty working group's strategy to represent the interests of the paediatrician within the clinical terms project.

\section{Child health specialty working group}

The child health specialty working group within the clinical terms project was established in January 1993. The group's membership evolved from the BPA Diagnostic Code Working Party and consists of seven paediatric advisers, one adviser from general practice, one adviser from paediatric nursing, and two salaried research workers. There is also a separate specialty assurance team whose job it is to provide an independent assessment of the group's output. This group has had to undertake a formidable list of tasks in order to ensure that terms used in the care of children are incorporated into the final product of the clinical terms project.

These tasks centred on ensuring that terminology describing diagnoses, procedures and investigations, peculiar to paediatrics and child health, were incorporated into the final thesaurus. This was very detailed work. It included creating a whole section of terminology to describe processes of community based paediatric practice, as well as detailed reviews of terms related to neonatology, paediatric surgery, and inborn errors of metabolism.

\section{Read clinical term structure}

Central to these tasks is the structure of the Read clinical term and the hierarchy within which they are stored. The adoption of a term for the Read thesaurus is a multistage process. The term describing a health entity must be identified, preferred terms and synonyms should be agreed and then organised into a file structure suitable for computerisation and cross referencing to internationally accepted classifications. The Read thesaurus consists of a structured list of preferred terms for each concept. The listing of these terms is within a hierarchical structure. At present there are five levels of hierarchy. This allows users of the software system to find terms and browse up and down the hierarchy to review related terminology (see table).

In order to ensure that the thesaurus of terms is able to describe the full width of clinical concepts, the need arose to allow separate qualifying terms to be linked to a Read term. This facility avoids the need for very long lists of mutually exclusive alternatives. An example is shown below:

\section{Structure of terms and additional detail \\ Core term:}

Removal of foreign body from bronchus. Qualifiers:

Site of foreign body: right main bronchus/left main bronchus.

Instrument used: rigid bronchoscope/ fibreoptic bronchoscope.

\section{When will it be available?}

At the time of writing, the child health specialty working group has three months to go before it will be disbanded and the NHS CCC takes over the process of collation and compiling of the specialty working group lists for incorporation into the Read clinical terms data set. The new terms created by the child health specialty working group will be included in the October 1994 release of Read clinical terms. They will continue to be updated within the planned maintenance programme. The NHS CCC has promised to provide a paediatric subset of Read clinical terms by October 1994 which will reduce the size of lists from which terms will be selected by paediatricians. It is planned that child health advisers will be linked to a maintenance programme whose role it would be to continue the development of Read clinical terms so that they continue to reflect everyday clinical practice in child health.

\section{How does this affect the paediatrician?}

It is hoped that the contribution of the child health specialty working group to this project will form a firm foundation for ensuring that the computerised language of health care within Read clinical terms will reflect everyday practice of child health. Whether this tool will improve access to health care information for the paediatrician, the audit specialist, or clinical researcher is very dependent upon factors outside the remit of the clinical terms project. The usability of the Read codes will depend upon the inclination of individual practitioners to adapt to the use of computerised techniques within their every day practice. Furthermore, it will be heavily dependent upon the system qualities of developments in information technology as well as the skill and imagination of the software authors in striving to automate the process of coding the medical record. National projects such as the integrated clinical workstation project are underway and hold promise for suggesting solutions. The 
development of computerised text and voice recognition, as well as increasingly sophisticated graphical interfaces will allow doctors to collect coded clinical data more easily. As the clinical terms project has enjoyed an almost unique cooperation between the branches of the medical profession, Read clinical terms and their codes are likely to be central to these developments.
1 Information Management Group. IM+T strategy overview. Leeds: NHS Management Executive, 1992.

2 Williams J, Morgan JM, Howlett PJ, Severs MP. Designing clinical information systems. BMF 1993; 305: 1435.

3 Buckland R. Language of health. BMF 1993; 306: 287-8.

4 Information Management Group. A national thesaurus of clinical terms in Read codes. London: HMSO, 1993 (3/93).

5 Information Management Group. What are Read codes?

London: HMSO, 1993 (4/93).
6 Information Management Group. Read codes and the terms project: a brief guide. London: NHS Management 